\title{
LA FORMACIÓN DE MAESTROS EN EDUCACIÓN INFANTIL DESDE LA PERSPECTIVA DE LA SOSTENIBILIDAD. UN ESTUDIO DE CASO EN LA UNIVERSIDAD DE CÁDIZ
}

\author{
Esther García-González \\ Rocío Jiménez-Fontana \\ Pilar Azcárate \\ Universidad de Cádiz
}

\section{RESUMEN}

La formación de los futuros maestros debe abarcar, además de cuestiones teórico-técnicas, habilidades y actitudes que los capaciten hacia la construcción de soluciones sostenibles para un mundo mejor. La investigación que presentamos analiza las prácticas docentes universitarias para conocer el grado de inclusión de la sostenibilidad en la Universidad de Cádiz, en concreto en el Grado de Maestro en Educación Infantil, a través del análisis de la metodología que pone en juego el profesor objeto de estudio. Se emplearon como instrumentos para la recogida de datos la técnica de observación participante bajo un guion de observación, el registro audiovisual y un diario de investigación. El análisis de datos se realizó a través de un sistema de categorías de elaboración propia. Los resultados caracterizan un escenario donde las estrategias metodológicas puestas en juego promueven en gran medida los principios de la sostenibilidad y, por tanto, el grado de integración de la sostenibilidad es elevado.

Palabras clave: Educación para la Sostenibilidad, Educación Superior, Metodologías, Aula Universitaria y Formación de Profesorado

\section{THE TRAINING OF CHILDHOOD EDUCATION TEACHERS FROM SUSTAINABILITY PERSPECTIVE. A STUDY CASE IN THE UNIVERSITY OF CÁDIZ}

\section{Abstract}

The training of future teachers cannot focus only in disciplinary knowledge, this needs to consider the skills and attitudes in order to empower to the students for design sustainable solutions for a better world. This research analyses universities teaching methodologies for to determine the degree of inclusion of sustainability in a classroom at the University of Cadiz, specifically on the Degree in Early Childhood Education, through the analysis of methodologies that the professor under study brings into play. We used the participant observation technique. In order to collect information two different instruments were employed, video record, checklist and a research diary. The analysis of the collected information was performed using a system of categories, of own elaboration. The achieved results reveal a scenario where the strategies methodological employed by the professor largely foster the sustainable principles. In the analysed case, we detected a high degree of integration of sustainability.

KeYwords: Education for Sustainable Development, Higher Education, Teaching Methods, University Classrooms and Teachers Training.

DOI: http://doi.org/10.25145/j.qurricul.2018.31.002

Revista QurRICUlum, 31; junio 2018, pp. 31-56; ISSN: e-2530-8386 


\section{LA CRISIS SOCIOAMBIENTAL COMO PUNTO DE PARTIDA}

La ciudadanía planetaria está sumergida en una grave crisis socioambiental, innegablemente ligada al uso utilitarista que una parte de la humanidad viene ejerciendo sobre el entorno. Una relación dominante del hombre sobre la naturaleza que ha provocado una degradación ambiental y social profunda. Esta situación se agrava cada día, pues el sistema capitalista imperante se ocupa de promover una cultura reduccionista y simplificadora que nos distrae, mientras se suceden acontecimientos de consecuencias probablemente irrevocables.

Nos enfrentamos a una crisis del conocimiento y del modelo de civilización, de dimensiones planetarias, que no puede ser tratada desde aspectos parciales sino que debe ser gestionada integralmente y enfocada tanto a las causas como al futuro (Leff, 2006). Es necesario sustituir la cultura de la simplificación causa-efecto por la cultura de la complejidad, en la que se reconozca la interrelación y la contingencia de todos los fenómenos (Morin, 1994). De manera que se impulse la creatividad hacia la gestación de redes de soluciones que favorezcan la armonización del hombre con la biosfera.

En este contexto urge buscar multisoluciones combinadas desde diversidad de ámbitos. Todas las disciplinas y quehaceres deben responder coordinadamente al reto ambiental de nuestros días (Puig, Echarri y Casas, 2014).

La Educación para la Sostenibilidad (en adelante ES) por su naturaleza trasformadora puede contribuir hacia esta convergencia entre campos de conocimiento; para ello, es imprescindible que permee al ámbito universitario (Barrón, Navarrete y Ferrer-Balas, 2010). Se trata no solo de incluir en las aulas universitarias contenidos relacionados con la sostenibilidad, hecho que simplificaría sobremanera su condición transdisciplinar (Wals y Jickling, 2002), sino más bien de cambiar formas de pensar, de actuar y de vivir dentro del mundo universitario y en concreto del espacio aula. El reto estriba en diseñar estrategias metodológicas que promuevan la evolución hacia una comprensión compleja del mundo. Lo cual confirma la responsabilidad de los profesores universitarios, que deben promover un espacio donde los estudiantes desarrollen un fuerte interés por la sostenibilidad (Michavilla, 2005).

Desde esta perspectiva, asumimos nuestra responsabilidad como investigadores del ámbito educativo, asiendo esta situación de crisis como coyuntura para acometer los cambios que demandan los procesos de enseñanza-aprendizaje universitarios.

En este trabajo, presentamos una investigación que aborda las metodologías docentes universitarias y su relación con la inclusión de la ES en sus aulas. Se centra en un caso de interés, un profesor del Grado de Maestro en Educación Infantil. Este trabajo forma parte de una investigación más amplia que comprende dos casos más, un profesor del Grado de Ciencias del Mar y Ambientales y otro del Grado de Grado de Contabilidad y Finanzas, los cuales han sido objetos de publicación (García-González, Jiménez-Fontana, y Navarrete, 2017; García-González, Jiménez-Fontana, Navarrete, y Azcárate, 2015).

La formación de maestros es un ámbito especialmente sensible, pues estos tendrán la responsabilidad en su actividad profesional de contribuir a la formación 
de la ciudadanía actual y futura, que debe estar preparada para los retos que plantea la situación de emergencia planetaria. Ello conlleva apostar por una formación que no se preocupe solo de lo científico-técnico, sino que capacite a los futuros maestros para comprender la complejidad de las interacciones de nuestro mundo y sus problemas conexos y a su vez los dote de recursos necesarios para la acción. En definitiva, hablamos de una educación integral que los prepare para la vida, el ejercicio de su profesión (Álvarez, 2012) y los ayude a construir una perspectiva hacia la sostenibilidad.

Trabajar en esta dirección implica serios desafíos a la comunidad docente; de una parte la reta a construir nuevas formas de hacer en el aula que atiendan a la emergencia de Educar para la Sostenibilidad, idea que confluye con el marco del Espacio Europeo de Educación Superior, que aboga por impulsar la incorporación de nuevas formas de trabajo en las aulas (Rodríguez Izquierdo, 2007). De otra, la emplaza a liderar el cambio en la universidad, ya que existe consenso en considerar al profesor como pieza clave en el éxito de cualquier innovación curricular (Cotton, Warren, Maiboroda y Bauley, 2007).

\section{SOLUCIONES DESDE EL ÁMBITO EDUCATIVO UNIVERSITARIO}

Si bien es cierto que afrontar la compleja situación que atraviesa nuestro planeta requiere la confluencia de actuaciones a diferentes escalas, qué duda cabe de que la educación y en concreto la ES juega un papel crucial en este entramado, es una herramienta clave para afrontar los retos a que se enfrenta la humanidad (UNESCO, 2015). A pesar de ello, estudios recientes revelan que la problemática de la sostenibilidad tiene una presencia insuficiente en la enseñanza (Vilches y Gil Pérez, 2016) y por ende en el ámbito universitario. Se han hecho avances en la integración de la formación para la sostenibilidad en los contenidos curriculares universitarios; sin embargo, están más centrados en el qué enseñar que en el cómo enseñar (Thomas, 2016), eje central de esta investigación.

La universidad debe formar profesionales desde el punto de vista científico, pero también debe prepararlos para emplear los conocimientos en la resolución de los problemas socioambientales, tomando decisiones y realizando intervenciones acordes con la sostenibilidad (Bravo, 2012).

A nuestro modo de ver, la ES constituye un pilar fundamental en la construcción de soluciones. Hablamos de ES como un proceso encaminado a adquirir claves para interpretar el mundo. Debe favorecer una construcción del conocimiento que abandone la consecución del pensamiento único y se encamine hacia una visión sistémica y compleja del funcionamiento del mundo, lo que se conoce como educación global (Flor, 2002). En definitiva, la ES propone un cambio desde una visión mecanicista y conductista hacia otra compleja, holística y crítica (Bonil et al., 2012) que invita a la capacitación para la acción (Breinting, 1997). Se postula como vía de preparación de ciudadanos capaces de reconocer, entender y solucionar las problemáticas socioambientales de su tiempo. En todo ello la universidad y particularmente sus profesores, como capacitadores de futuros profesionales (Walker, 2016), tienen una responsabilidad ineludible (Ull, 2011). 
Emerge desde esta perspectiva un reto para este colectivo, transformar los procesos educativos para dar respuesta a las necesidades que demandan los tiempos actuales (García et al., 2016). Unos tiempos impregnados de una crisis global, cuyo entendimiento requiere de una perspectiva compleja que permita atender a la realidad desde la máxima diversidad posible (De la Herrán Gascón, 2005). Una forma de ver el mundo en la cual se tenga en cuenta el tejido de «acciones, interacciones, retroacciones, determinaciones, azares, que constituyen nuestro mundo fenoménico» (Morin, 1994, p. 32), los cuales permitan proponer soluciones fundamentadas en la sostenibilidad, para luchar por un mundo mejor.

Respuesta que, en gran medida, está relacionada con la inclusión de los principios de la sostenibilidad en el ámbito universitario. Principios definidos por la CADEP-CRUE ${ }^{1}$ (2012) como principio ético, holístico, de complejidad, glocalización, transversalidad y responsabilidad social universitaria. La inclusión de estos principios conlleva cambios en la concepción del proceso de enseñanza-aprendizaje (Vilches y Gil Pérez, 2012) más aún cuando se está formando maestros responsables de la educación de las generaciones futuras.

Las reflexiones que desde hace tiempo forman parte de las discusiones de nuestro grupo de investigación Desarrollo Profesional del Docente "DPD» giran en torno a cómo integrar la sostenibilidad en nuestras aulas desde el sentido transformador que contiene la misma. Trabajar desde esta perspectiva conlleva unas determinadas formas de hacer, unas prácticas docentes acordes con los principios de la sostenibilidad. Apunta a focalizar el sentido de la práctica docente en aquello que tiene que ver directamente con cómo se enseña, es decir, las actividades, su diversidad, su relación con el proceso de aprendizaje, los conocimientos puestos en juego, los recursos, la evaluación... El análisis de la misma práctica constituye un elemento básico para conocer el nivel de desarrollo profesional de los profesores universitarios (García et al., 1999).

Desde estas ideas caracterizamos unas líneas metodológicas que consideramos pueden favorecer la inclusión de la sostenibilidad en las aulas universitarias y que sirven como referente de análisis para esta investigación. Son fruto de la revisión bibliográfica y de estudios precedentes en este campo (Cardeñoso, Azcárate, y Oliva, 2013). Estas líneas metodológicas respetan la complejidad de los procesos de enseñanza-aprendizaje, respetando la coexistencia en el aula de ideas diversas, que consideramos indisolubles y complementarias (Morin, 2008). Estas ideas se articulan a través del diálogo entre extremos e interactúan con los agentes participantes del proceso, profesor, alumno y contenidos, configurando la acción educativa. El tratamiento en el aula de estas líneas y su concreción en acciones coherentes con la sostenibilidad permiten caracterizar los procesos de enseñanza-aprendizaje. Las líneas metodológicas que proponemos se concretan en ejes dialógicos, descritos a

${ }^{1}$ CADEP-CRUE: Calidad Ambiental, Desarrollo Sostenible y Prevención de Riesgos-Conferencia de Rectores de las Universidades Españolas. 
continuación y más desarrollados en anteriores publicaciones (García-González, Jiménez-Fontana, Azcárate, \& Cardeñoso, 2016):

\section{- Relación profesor-alumno: Vertical $\leftrightarrow$ Horizontal}

La perspectiva vertical asume al docente como responsable del proceso de enseńanza-aprendizaje y la horizontal dota a cada individuo de un rol activo dentro del proceso (Viladot y Pedreira, 2012). La integración de la sostenibilidad configura un proceso donde el docente se comporta como el mediador que pone en juego las herramientas de aprendizaje y donde la responsabilidad de lo que ocurre en el aula es compartida entre alumnos y profesor.

\section{- Competencias: Especificas $\leftrightarrow$ Transversales}

Las competencias para la sostenibilidad deben capacitar al alumnado para hacer frente a las problemáticas socioambientales preparándolo para hacer valoraciones complejas de su trabajo y el ajeno, y tomar decisiones en las circunstancias impredecibles que encontrará en el futuro (Rieckmann, 2012). La competencia específica define los conocimientos clave de la disciplina y la transversal vincula el contenido al medio. La sostenibilidad permite integrar ambos tipos de competencias.

\section{- Realidad socioambiental: No integrada↔Integrada}

Implicar en el aula la realidad socioambiental fomenta en los estudiantes la autonomía, la responsabilidad y la capacidad de compromiso. Muestra que se puede abordar el conocimiento desde caminos diversos y que existen varios medios para solucionar una misma problemática. Se deben establecer puentes entre esta y el conocimiento disciplinar, facilitando la perspectiva sistémica y transdisciplinar propia de la sostenibilidad (Wals y Jickling, 2002).

\section{- Recursos: Internos $\leftrightarrow$ Externos}

Es necesario combinar el uso de recursos internos cuya función es organizar el proceso de enseñanza-aprendizaje, con el uso de los recursos existentes en nuestro entorno (diálogos con expertos, salidas de campo, intervenciones directas, afrontar problemáticas socioambientales, prácticas en centros específicos, etc.). Se ha de conseguir que el entorno entre en el aula (Wiek et al., 2014) y el aula salga de la universidad. Romper las barreras del aula y trabajar con la comunidad y con el entorno cercano en sentido amplio significa establecer espacios socioeducativos en y para la sostenibilidad. Se trata de utilizar todos los recursos de que disponemos, potenciando las sinergias que se producen al compaginarlos para promover la sostenibilidad.

\section{- Dinámicas de aula: Cerradas $\leftrightarrow$ Abiertas}

Las dinámicas cerradas son necesarias para ordenar ideas, asentar bases, dar orientaciones. Deben alternarse con dinámicas abiertas que permitan la inclusión de nuevos contenidos, centros de interés o problemas. El profesor debe estimular la 
formulación de preguntas, ofrecer nuevas visiones y poner de relieve la curiosidad como estímulo para construir conocimiento. Una dinámica de aula coherente con los principios y valores de la sostenibilidad supone dotar de voz a los alumnos, para que negocien y tomen partido en clase. La idea es capacitar a los estudiantes para tomar decisiones de forma autónoma, fundamentada y responsable y así caminar hacia la construcción de una ciudadanía crítica, participativa y transformadora (Mata-Benito, Ballesteros-Velázquez y Padilla-Carmona, 2013).

\section{- Trabajo de aula: Individual En grupo}

La aproximación a la información es en primer término individual, es el contraste con el grupo lo que provoca el conflicto (Coll, 1994). Este conduce a una reflexión y reestructuración de las ideas, haciéndose posible el aprendizaje a través del diálogo activo con el resto del grupo; este aprendizaje se afianza posteriormente con una reconstrucción personal. La sinergia entre ambas estrategias aporta significado al aprendizaje. Desde la sostenibilidad, esta combinación promueve la formación integral del individuo, recreando situaciones que los alumnos encontrarán durante su vida profesional y personal. Se trabaja la responsabilidad de pertenencia al grupo, se favorece la comunicación, se ponen en marcha estrategias de cómo aprender, criticar y abordar el conocimiento, mediante negociación de significados.

\section{- Evaluación: Acreditativa $\leftrightarrow$ Procesual}

La evaluación acreditativa es una evaluación final, la constatación ante la sociedad de los aprendizajes alcanzados. La procesual recoge información sobre proceso y participantes, los resultados tienen repercusiones sobre ambos (Sanmartí, 2007). Considera aspectos cognitivos, afectivos y de acción. Ambas funciones son complementarias, una es necesaria por el contexto legal y la otra para regular el aprendizaje-enseñanza. La evaluación entendida como reflexión, valoración y elemento de mejora es un componente esencial para afrontar la complejidad que suponen los problemas socioambientales a los que se enfrentarán los alumnos en su cotidianidad y en el mundo laboral.

La combinación de estas líneas metodológicas en la puesta en práctica del aula y su interacción entre los agentes profesor, alumno y contenidos permitirá emerger el grado de acercamiento de las prácticas docentes a los principios de la sostenibilidad. Servirán asimismo como elemento de contraste para la investigación que presentamos.

El objetivo del proceso formativo en el caso del Grado de Educación Infantil es formar agentes de cambio, profesionales, maestros en este caso, capaces de transformar su realidad construyendo un entorno sostenible; para ello no es viable cualquier forma de intervención en el aula. Consideramos que solo aquello que se ha practicado de manera habitual puede ser asumido y transferido a otros contextos, las formas de hacer en el aula se convierten así en parte del contenido a aprehender. En este sentido es importante la inclusión en las aulas universitarias de estrategias metodológicas que incidan en una ES, caracterizarlas es el objeto de este trabajo. 


\section{DISEÑO Y METODOLOGÍA DEL ESTUDIO}

La finalidad de nuestra investigación es analizar en qué medida la metodología desarrollada en el aula puede ser una vía para incluir la sostenibilidad en los procesos formativos universitarios.

Para acceder a dicha información, formulamos dos cuestiones que orientan la investigación:

- Caracterizar el proceso de enseńanza-aprendizaje que se desarrolla en el aula estudiada.

- Analizar el grado de inclusión de sostenibilidad implícito en las estrategias metodológicas utilizadas.

Para ello ha sido necesario construir un instrumento de análisis que nos ha permitido tratar los datos obtenidos y caracterizar las estrategias puestas en juego. El estudio realizado nos permitirá comprobar la utilidad de dicha herramienta de análisis de datos para conocer la presencia de la sostenibilidad en las aulas universitarias.

La naturaleza del ámbito de estudio conduce hacia un enfoque cualitativo, el cual determina la correspondencia entre el proceso de investigación y los supuestos teóricos de forma flexible, adaptándose a la realidad que se investiga (Hernández, Fernández y Baptista, 2008).

Optamos por un estudio de caso, centrado en un caso que presenta interés por sí mismo (Stake, 2005). El profesor objeto de estudio trabaja en un ámbito del conocimiento al cual se le presupone implicación en el ámbito de la sostenibilidad, Ciencias de la Educación. Posee experiencia en el ámbito de la ES, ha participado en un proyecto de innovación docente en este sentido y su línea de investigación se dirige hacia la mejora de los procesos de enseñanza-aprendizaje y la puesta en práctica de estrategias que intensifiquen la conexión de la universidad con el ámbito social.

A estos aspectos, se suma el interés que despierta el ámbito profesional del sujeto, la formación inicial del profesorado de Educación Infantil.

El contexto del estudio es una asignatura de segundo curso del Grado de Maestro en Educación Infantil, pertenece al ámbito de la atención a la diversidad, cuenta con 68 alumnos matriculados.

\subsection{InSTRUMENTOS DE RECOGIDA DE DATOS}

Para la toma de datos empleamos fundamentalmente la observación participante. En concreto los instrumentos utilizados para la recogida de información fueron el registro audiovisual (codificado como I-1) y un diario de investigación (codificado como I-2). En dicho diario se anotaron acontecimientos, impresiones y valoraciones de interés para la investigación, apoyado en un guion de observación que permitió centrar la observación en los aspectos de interés para la investigación. Este fue previamente validado dentro del grupo de investigación DPD. 
Durante el curso se realizó un seguimiento de la evolución de la asignatura con la presencia en el aula de la investigadora. También se grabaron nueve sesiones de 60 minutos distribuidas a lo largo del curso y previamente pactadas con el profesor, se combinaron clases teóricas y prácticas, para realizar un análisis más pormenorizado.

\subsection{INSTRUMENTOS Y TÉCNICAS DE ANÁLISIS DE DATOS}

Como hemos indicado, para el análisis de datos diseńamos un sistema de categorías e indicadores que configuró la Herramienta para el Análisis Metodológico desde la Sostenibilidad (HAMS). Su elaboración está basada en la fundamentación teórica de la investigación y antes de su implementación fue sometida a un proceso de validación aparente y de contenido (Litwin, 1995). La HAMS se configura a partir de los tres agentes clave que interactúan en el aula, profesor-alumno-contenidos, y las líneas metodológicas puestas en juego, definidas en el epígrafe anterior, que permiten caracterizar las formas de hacer del docente. La relación de la práctica docente con la sostenibilidad es un emergente resultante del análisis de resultados.

De esta forma, las líneas metodológicas constituyen las diferentes categorías de análisis, que denominamos ejes dialógicos, los cuales quedan descritos a partir de un conjunto de indicadores formulados en gradación. Cada indicador se corresponde con una posible decisión del profesor en relación con la gestión del eje dialógico al que hace referencia, según sean más o menos cercanas a la inclusión de la sostenibilidad.

Definimos tres tipos de gradientes en función de los indicadores que describen el eje dialógico en cuestión, estos gradientes van de estadios simples a complejos pasando por una zona de tránsito. Cada estadio hace referencia a distintos grados de integración de la sostenibilidad en el aula. El gradiente 1 contiene cinco indicadores; el gradiente 2 posee cuatro indicadores; y el gradiente 3 , tres indicadores. De manera que la zona de tránsito permite tres estadios (inicial, medio y avanzado) para el gradiente 1 , dos (inicial y avanzado) para el gradiente 2 y uno (medio) para el gradiente 3, como refleja la figura 1 :

\begin{tabular}{lccccc} 
& \multirow{2}{*}{ SIMPLE } & INICIAL & MEDIO & AVANZADO & COMPLEJO \\
\cline { 2 - 5 } & & \multicolumn{3}{c}{ ZONA DE TRÁNSITO } \\
\hline Gradiente 1 & 1 & 2 & 3 & 4 & 5 \\
\hline Gradiente 2 & 1 & 2 & 3 & 4 \\
\hline Gradiente 3 & 1 & & 2 & & 3 \\
\hline
\end{tabular}

Fuente: elaboración propia.

Figura 1. Gradiente de indicadores.

La HAMS, incluyendo los indicadores referidos a los tres agentes, se presenta en las tablas 1,2 y 3. 


\section{Profesor}

1. El profesor es el único agente que elige y dirige el proceso de enseńanza-aprendizaje.

2. El alumno adquiere cierto protagonismo en el proceso de enseńanza-aprendizaje, pero es el profesor quien lo dirige y lidera.

3. El profesor pone en juego un proceso de enseńanza-aprendizaje considerando nuevos contenidos e inquietudes que se trabajan momentáneamente, sin cambiar sustancialmente su planificación inicial.

4. Las reacciones del profesor ante las intervenciones de sus alumnos facilitan la participación y estos adquieren un mayor protagonismo.

5. El profesor se comporta como un mediador/facilitador en el proceso de enseñanza-aprendizaje, de manera que la dinámica de clase es abierta a todos los participantes.

\footnotetext{
: profesionales de la disciplina que estudian.

2. Existen insinuaciones, pero no de forma directa o clara, sobre el compromiso de los alumnos en el desarrollo de su profesión.

3. Se hacen referencias a la responsabilidad como profesionales en la resolución de problemas que tendrán los alumnos.
}

1. El discurso no incluye la realidad socioambiental, se circunscribe a las temáticas de la asignatura.

2. El discurso incluye algunos aspectos sobre realidad socioambiental, pero ligada preferentemente a la dimensión más próxima a su disciplina, y no contempla las interacciones entre las mismas.

3. En el discurso se impulsan valores ciudadanos y la participación social; abordándose las posibles interrelaciones entre lo social, lo económico y lo ambiental.

4. Se ponen en juego y analizan diferentes posicionamientos éticos ante la realidad socioambiental.

5. Se abordan interrelaciones entre lo social, lo ambiental y lo económico, se potencian las aportaciones de diferentes disciplinas y los enfoques de otros ámbitos de conocimiento desde una perspectiva interdisciplinar o multidisciplinar.

1. Las clases se desarrollan con el empleo de recursos de contexto interno, al margen del contexto externo.

2. Se refiere la existencia de recursos externos, pero como mera información a considerar.

3. Se admiten abiertamente las posibilidades de intervención en la problemática ambiental, pero sin animar a una participación activa.

4. Se promueve y anima la intervención en la problemática socioambiental del entorno.

5. Se trabaja con recursos de orden interno y externo indistinta y conjuntamente, animando a la interacción con el entorno.

1. Las preguntas/estrategias planteadas tienen una respuesta única.

2. Aunque se valoran diferentes respuestas a los interrogantes planteados, solo se considera acertada la prevista por el profesor.

3. Fórmula pregunta/estrategias como medio para conocer qué interesa a los alumnos, reorientar la actividad y dar una u otra información.

4. Se potencian y valoran las respuestas divergentes y diversas a una misma pregunta/estrategia sin que exista una respuesta única prevista. 
1. El alumno en clase solo toma apuntes y posteriormente prepara individualmente las pruebas o exámenes finales, sin interactuar con los compańeros.

2. Se facilita la participación unidireccional y vertical en clase.

3. Se trabaja tanto individual como grupalmente, en clase y fuera de ella, potenciándose el trabajo en equipo. Se tienen en cuenta los intereses del colectivo y los individuales.

4. El trabajo se organiza cooperativamente entre los participantes, debiendo tomar decisiones como colectivo y coordinado con dinámicas individuales.

1. Se evalúa al alumno a través de pruebas o exámenes parciales o finales.

2. Se utiliza, además del examen, trabajos individuales, participación en clase, implicación..., que se centra, solo sobre el alumno.

3. Se utilizan diferentes elementos de evaluación por el profesor y se tiene en cuenta la del alumno: autoevaluación, co-evaluación, en distintos momentos del proceso.

4. La evaluación se realiza durante todo el proceso con diferentes instrumentos, participan profesor y alumno y la información obtenida redunda sobre la marcha del proceso.

Fuente: elaboración propia.

TABLA 2. HAMS PARA AGENTE ALUMNO

Alumno

Categorías INDicadores

1. La participación de los alumnos es dirigida y mediatizada por el profesor.

2. Los alumnos intervienen y participan durante el desarrollo de las clases de forma espontánea, sin esperar la invitación del profesor.

3. Los alumnos pueden expresar sus opiniones sobre el proceso pero es el profesor quien toma la última decisión.

4. Los alumnos participan en el proceso activamente, pero no se profundiza en esta participación, sino que se reconduce hacia la planificación inicial.

5. La participación de los alumnos forma parte del propio desarrollo del proceso de enseńanza-aprendizaje, siendo los protagonistas del proceso y el profesor un mediador. Se promueve la participación democrática en la resolución de conflictos de aula y en la toma de decisiones.

1. La realidad socioambiental no se refleja en el desarrollo de las clases y, por tanto, no se liga con el papel profesional de los alumnos.

2. En ocasiones, se relaciona la realidad socioambiental y el papel profesional que desarrollarán los alumnos.

3. La realidad socioambiental es inherente al papel y desarrollo profesional de los alumnos y así se manifiesta en las clases (a través del discurso, actividades...).

1. No se tienen en cuenta los intereses e inquietudes de los alumnos, solo la planificación inicial.

2. Se atienden los intereses de los alumnos, dando espacios para la reflexión, pero no se desarrollan las propuestas.

3. Se asumen propuestas de los alumnos en relación con la formulación y tratamiento de problemas, de contenidos transversales, de actividades... Se reorganiza el proceso y se promueve la reflexión y el análisis de las propuestas en la dinámica de aula. 


\section{Alumno}

Fuente: elaboración propia.

1. El trabajo en el aula individual es el recurso más utilizado. El trabajo en grupo no forma parte de las estrategias metodológicas.

2. Hay trabajo en grupo de forma esporádica, es solo un recurso metodológico no significativo.

3. El trabajo en grupo es significativo y tiene un papel relevante en el proceso.

4. Se combina el trabajo cooperativo con el individual durante el desarrollo de las clases y ambos son significativos en el proceso de enseñanza-aprendizaje.

\section{TABLA 3. HAMS PARA AGENTE CONTENIDOS}

\begin{tabular}{ccc} 
Contenidos \\
\hline CATEgorías
\end{tabular}

4. Cuando se abordan los contenidos solo se trabajan conocimientos propios de la materia.

5. Se tratan esporádicamente, además de los conocimientos de la materia, aspectos no estrictamente disciplinares y con referencias a la realidad socioambiental.

6. Se abordan conocimientos de otras áreas o disciplinas de manera transversal, de forma que la realidad socioambiental subyace y articula su tratamiento.

7. No se utilizan problemáticas socioambientales para abordar los contenidos.

8. Se hacen referencias a problemáticas, pero no se utilizan como recurso para trabajar los contenidos.

9. Se utilizan casos reales para implicar los contenidos haciendo referencias a las problemáticas actuales. (Se da aplicabilidad a los contenidos).

10. Las preguntas/estrategias realizadas son finalistas y solo buscan reafirmar los contenidos que se están impartiendo.

11. Se abren nuevos interrogantes que hacen reflexionar a los alumnos, pero no se usan sus respuestas como estrategia para abordar los contenidos.

12. Las preguntas/estrategias que se formulan son abiertas, desarrollándose dinámicas donde las propias respuestas se convierten en contenido y se emplean para profundizar y avanzar sobre ellos.

Fuente: elaboración propia. 
La codificación de los indicadores que configuran la HAMS se realizó como se muestra en la tabla 4. Presentamos un ejemplo para el agente profesor (P), codificación que se repite para los agentes contenidos (C) y alumno (A), en el que se indica el agente y el eje dialógico. En las tablas siguientes a esta, al final del código se indica la numeración correspondiente a cada indicador que va reflejando el estadio de integración de sostenibilidad.

\begin{tabular}{|c|c|c|c|}
\hline \multicolumn{4}{|c|}{ TABLA 4. CÓDIGOS DE LOS INDICADORES PARA EL AGENTE PROFESOR } \\
\hline AGENTE & Código & EJE DIALÓGICO & CÓDIGO FINAL \\
\hline \multirow{7}{*}{$\begin{array}{l}\grave{D} \\
\stackrel{0}{0} \\
\stackrel{0}{0} \\
0\end{array}$} & \multirow{7}{*}{$P$} & Relación profesor-alumno, Vertical $\leftrightarrow$ Horizontal & PRVH \\
\hline & & Competencias, Específicas $\leftrightarrow$ Transversales & PCET \\
\hline & & Realidad socioambiental, No integrada $\leftrightarrow$ Integrada & PRNI \\
\hline & & Recursos, Internos $\leftrightarrow$ Externos & PRIE \\
\hline & & Evaluación, Acreditativa $\leftrightarrow$ Procesual & PEAP \\
\hline & & Dinámicas, Cerradas↔Abiertas & PDAC \\
\hline & & Trabajo individual $\leftrightarrow$ En grupo & PTIG \\
\hline
\end{tabular}

Fuente: elaboración propia.

Para su análisis, los datos fueron reducidos a través de la configuración de diferentes unidades de información con sentido en sí mismas, desde los principios del análisis del discurso (Pińuel, 2002). Estas unidades fueron clasificadas y codificadas a través de la HAMS.

El procesado informático de las unidades codificadas se realizó con el software de análisis cualitativo QRS N-Vivo9, que permite concretar las frecuencias de las unidades a través de sus códigos, combinando técnicas cualitativas con cuantitativas.

Para garantizar la fiabilidad de los datos usamos la triangulación y el contraste de perspectivas, fuentes y datos, dentro del grupo de investigación DPD (Bisquerra, 1989).

\section{RESULTADOS Y DISCUSIÓN}

Los resultados y su discusión se presentan en dos fases que dan respuesta a las dos cuestiones de investigación formuladas anteriormente. La primera corresponde a un análisis descriptivo sobre el proceso de enseñanza-aprendizaje del aula de estudio. En esta fase se caracteriza cada eje dialógico a partir de la integración entre los resultados obtenidos para los tres agentes profesor-alumno-contenidos. Entender la realidad del aula en su plenitud requiere analizar las interacciones que se producen entre los elementos que la configuran. Así, la conjunción de los tres agentes y los diferentes ejes dialógicos devuelve una imagen del proceso de ensenanza-aprendizaje analizado. 
La segunda fase analiza el grado de inclusión de la sostenibilidad en el aula. Este viene determinado por las frecuencias relativas de los diferentes indicadores y sus correspondientes estadios de integración de sostenibilidad (figura 1) contenidos en los ejes dialógicos para cada agente analizado, profesor-alumno-contenidos.

\subsection{FASE 1: CARACTERIZACIÓN DEL PROCESO DE ENSEÑANZA-APRENDIZAJE}

Presentamos los resultados para cada eje dialógico. Se muestra una tabla que recoge las diferentes frecuencias obtenidas por indicador, especificando el agente, profesor, alumno o contenido, al que pertenece. Seguidamente se da sentido a los resultados a través del significado de los distintos indicadores. Se exponen los resultados más significativos para ejemplificar los objetivos de la investigación. la tabla 5 .

Los datos relativos al eje dialógico Relación profesor-alumno se recogen en

\begin{tabular}{|c|c|c|c|}
\hline EJE DIALÓGICO & Agente & INDICADOR & FRECUENCIA RELATIVA \\
\hline \multirow{12}{*}{$\begin{array}{l}\text { Relación profesor-alumno, } \\
\text { Vertical } \leftrightarrow \text { Horizontal }\end{array}$} & \multirow{4}{*}{ Profesor } & PRVH1 & $11.8 \%$ \\
\hline & & PRVH2 & $50 \%$ \\
\hline & & PRVH4 & $11.8 \%$ \\
\hline & & PRVH5 & $26.4 \%$ \\
\hline & \multirow{5}{*}{ Alumno } & ARVH1 & $24 \%$ \\
\hline & & ARVH2 & $13.8 \%$ \\
\hline & & ARVH3 & $7 \%$ \\
\hline & & ARVH4 & $3.5 \%$ \\
\hline & & ARVH5 & $51.7 \%$ \\
\hline & \multirow{3}{*}{ Contenidos } & CRVH1 & $6.4 \%$ \\
\hline & & CRVH2 & $16.2 \%$ \\
\hline & & CRVH3 & $77.4 \%$ \\
\hline
\end{tabular}

Fuente: elaboración propia.

Los resultados obtenidos se distribuyen a lo largo de todo el gradiente de indicadores. Se ha identificado un proceso donde la participación de los alumnos es un eje básico para su funcionamiento [ARVH5 fi=51.7\%]; además, los contenidos están al servicio de la resolución de problemas, son un recurso más del proceso de enseñanza-aprendizaje [CRVH3 fi=77.4 \%]. Un ejemplo en este sentido: 
I-2_CRVH32: Durante el curso, los alumnos por grupos están inmersos en un proceso de investigación sobre un tema de la asignatura elegido por ellos. El profesor se centra en provocar nuevos interrogantes, proporcionar recursos y conectar la investigación con la asignatura.

Esta situación contrasta, en cierta medida, con el papel que desarrolla el docente que aun concediendo protagonismo a los alumnos, especialmente en las clases prácticas [PRVH5 $\mathrm{fi}=26.4 \%$ ], es quien dirige fundamentalmente el proceso, mediatizando la participación de los alumnos [PRVH2 fi=50\%; ARVH1 fi=24\%]:

I-1_PRVH2: Me gustaría pedir una voluntaria para construir los conceptos que veremos en clase, el otro grupo no lo ha hecho, con lo cual, vamos a llegar a una serie de conclusiones, las vamos a poner en el diario y veremos cómo se complementa con lo de nuestras compańeras.

Los datos reflejan una relación entre profesor y alumno marcada por la horizontalidad, lo cual indica desde la perspectiva de la ES que la responsabilidad de lo que ocurre en el aula es en parte compartida. La participación de los alumnos es el vector motor del desarrollo de las clases y los contenidos sirven de instrumento para la construcción del conocimiento. No obstante, contenidos y alumnos están condicionados por la actuación del profesor en el aula, que cede espacios de protagonismo al resto de agentes, pero es quien principalmente organiza y decide qué papel juegan los demás agentes.

Los datos relativos al eje dialógico Competencias se recogen en la tabla 6 .

\begin{tabular}{cccc}
\hline \multirow{2}{*}{ TABLA 6. EJE DIALÓGICO COMPETENCIAS: ESPECÍFICAS-TRANSVERSALES } \\
\hline Eje Dialógico & Agente & IndiCADor & FreCuenCia Relativa \\
\hline \multirow{2}{*}{$\begin{array}{c}\text { Competencias, } \\
\text { Específicas } \leftrightarrow \text { Transversales }\end{array}$} & \multirow{2}{*}{ Profesor } & PCET1 & $60.8 \%$ \\
\cline { 3 - 4 } & & PCET2 & $34.8 \%$ \\
\cline { 3 - 4 } & & PCET3 & $4.4 \%$ \\
\hline
\end{tabular}

Fuente: elaboración propia.

En el discurso del profesor no detectamos prácticamente alusiones al papel de agente de cambio que tendrán los alumnos como futuros profesionales, en este caso maestros, pues la mayoría de información se refiere a la materia que se imparte. Como vemos la frecuencia más alta se corresponde con el gradiente simple [PCET1 $\mathrm{fi}=60.8 \%]$ :

2 I-2_CRVH3: Diario de investigador (I-2)_Contenidos (C) Relación Vertical-Horizontal $(\mathrm{RVH})$ Estadio de integración de sostenibilidad medio (3). 
I-1_PCET133: Como decíamos ayer, hay que tener cuidado con las barreras en atención a la diversidad; fijaos, aquí tenemos una barrera ambiental, el mero hecho de que dé aquí el reflejo hace que una parte de la clase se pierde una información importante.

Aunque, en determinadas situaciones, sí emergen insinuaciones sobre el compromiso de los alumnos en el desarrollo de su profesión (gradiente medio) [PCET2 fi=34.8\%]. Como se extrae de la siguiente afirmación:

I-1_PCET2: Las etiquetas condicionan nuestro comportamiento con respecto a las personas y las etiquetas se aprenden, se aprenden socialmente. Con lo cual, si los maestros no ponemos etiquetas, nuestros nińos y nińas no aprenderán las etiquetas sino su diversidad.

Estos resultados indican que las competencias están más encaminadas al desarrollo de capacidades propias de la disciplina que a la formación en la resolución de problemas socioambientales que formarán parte del desarrollo profesional de los futuros maestros, aunque se hayan detectado algunos indicios en este sentido. Por tanto, competencias transversales, más en sintonía con los propósitos que persigue la ES, casi no tienen cabida en el aula. la tabla 7.

Los datos relativos al eje dialógico Realidad socioambiental se recogen en

\begin{tabular}{|c|c|c|c|}
\hline EJe dialógico & Agente & INDICADOR & Frecuencia RELATIVA \\
\hline \multirow{6}{*}{$\begin{array}{l}\text { Realidad socioambiental, } \\
\text { No integrada↔Integrada }\end{array}$} & \multirow{2}{*}{ Profesor } & PRNI1 & $52.4 \%$ \\
\hline & & PRNI2 & $47.6 \%$ \\
\hline & \multirow{2}{*}{ Alumno } & ARNI1 & $56 \%$ \\
\hline & & ARNI2 & $44 \%$ \\
\hline & \multirow{2}{*}{ Contenidos } & CRNI1 & $50 \%$ \\
\hline & & CRNI2 & $50 \%$ \\
\hline
\end{tabular}

Fuente: elaboración propia.

En relación con este eje, identificamos numerosos casos donde la realidad socioambiental no está integrada, las frecuencias relativas se ciñen a gradientes bajos. De forma que no hay vinculación de esta con el papel de los alumnos como futuros maestros [ARNI1 fi $=56 \%$ ]. El discurso y los contenidos suelen ir ligados a temas específicos de la materia [PRNI1 fi=52.4\%; CRNI1 fi=50\%]. Un ejemplo:

${ }^{3}$ I-1_PCET1: Registro audiovisual (I-1)_Profesor (P) Competencias Específicas-Transversales (CET) Estadio de integración de la sostenibilidad simple (1). 
I-1_PRNI1: Hemos concluido que también sigue siendo físico, psíquico o sensorial pero afecta a la persona, que puede nacer con ello o lo puede adquirir.

Sin embargo, no puede decirse que esta ausencia de la realidad socioambiental sea una tónica en el aula. Tal como indican las frecuencias detectadas, aunque con un valor ligeramente menor, se distinguen en el discurso del profesor temas relacionados con esta; por tanto, no estrictamente disciplinares [PRNI2 $\mathrm{fi}=47.6 \%$; CRNI2 fi $=50 \%$ ], o donde se vincula con el desarrollo profesional del alumno [ARNI2 fi=44\%]:

I-1_ARNI2: Está bien que este grupo enlazara el tema de la orfandad con la muerte, podían haber hecho otra cosa más sencilla para trabajar pero han profundizado en un elemento que normalmente en la escuela no se trabaja.

Los resultados muestran que la realidad socioambiental no es el eje principal de actuación. Sin embargo, aun no teniendo un grado de integración alto, sí que forma parte del proceso de enseñanza-aprendizaje. Desde la perspectiva de la ES este es un aspecto clave pues ayuda a conectar los contenidos disciplinares con la realidad donde los maestros ejercerán su profesión.

Los resultados para el eje dialógico Recursos se presentan en la tabla 8.

\begin{tabular}{cccc}
\hline \multirow{3}{*}{ TABLA 8. EJE DIALÓGICO RECURSOS: INTERNOS-EXTERNOS } \\
\hline \multirow{2}{*}{ EJE DIALógico } & Agente & INDICADOR & FreCUENCIA RELATIVA \\
\hline \multirow{3}{*}{$\begin{array}{c}\text { Recursos, } \\
\text { Internos } \leftrightarrow \text { Externos }\end{array}$} & Profesor & PRIE1 & $55.6 \%$ \\
\cline { 3 - 4 } & & PRIE2 & $44.4 \%$ \\
\cline { 3 - 4 } & Contenidos & CRIE1 & $25 \%$ \\
\cline { 3 - 4 } & & CRIE2 & $25 \%$ \\
\cline { 3 - 4 } & & CRIE3 & $50 \%$ \\
\hline
\end{tabular}

Fuente: elaboración propia.

En relación con el agente profesor y el desarrollo de las clases, se ha identificado que estas se sustentan principalmente en la explicación del profesor o algunos recursos audiovisuales [PRIE1 fi= 55.6\%], es decir, recursos de orden interno. Si bien es cierto que se incluyen recursos de orden externo como información a considerar en el proceso [PRIE2 $\mathrm{fi}=44.4 \%]$ :

I-1_PRIE2: ¿Os habéis apuntado a las jornadas de Atención a la Diversidad que os comenté?

Asimismo, en determinadas situaciones, se usan casos reales para implicar los contenidos. Incluso se relacionan con ejemplos de la propia aula [CRIE3 fi=50\%]. Un ejemplo del caso que exponemos: 
I-1_CRIE3: Esta tarima no es accesible, le faltaría una rampa, para que las personas con movilidad reducida pudiesen subir. También le falta el borde delimitado con una cinta amarilla, para una persona que tenga un déficit visual. La accesibilidad tiene que ver con adaptar los espacios a todas las personas.

No obstante, los datos reflejan una situación no constante en el aula, pues también se han identificado ejemplos en los cuales no se usan estas problemáticas como recurso [CRIE1 fi=25\%] y otros en los que aunque se tienen en cuenta son simple información que no se usa como recurso [CRIE2 fi=25\%].

Por tanto, encontramos una situación en la que la balanza se inclina levemente hacia el uso de recursos internos, aunque se combinan con los externos. Es necesario desde la idea de ES que defendemos utilizar recursos externos de manera que los muros de las aulas se difuminen y estas se hagan más transparentes a la sociedad. en la tabla 9.

Para el caso de las Dinámicas de aula, los resultados obtenidos se presentan

\begin{tabular}{|c|c|c|c|}
\hline \multicolumn{4}{|c|}{ TABLA 9. EJE DIALÓGICO DINÁMICAS DE AULA: CERRADAS-ABIERTAS } \\
\hline EJE dialógICo & Agente & INDICADOR & FRECUENCIA RELATIVA \\
\hline \multirow{8}{*}{$\begin{array}{l}\text { Dinámicas de aula, } \\
\text { Cerradas } \leftrightarrow \text { Abiertas }\end{array}$} & \multirow{3}{*}{ Profesor } & PDAC2 & $8.3 \%$ \\
\hline & & PDAC3 & $50 \%$ \\
\hline & & PDAC4 & $41.7 \%$ \\
\hline & \multirow{2}{*}{ Alumno } & ADAC2 & $92 \%$ \\
\hline & & ADAC3 & $8 \%$ \\
\hline & \multirow{3}{*}{ Contenidos } & CDAC1 & $5.6 \%$ \\
\hline & & CDAC2 & $33.3 \%$ \\
\hline & & CDAC3 & $61.1 \%$ \\
\hline
\end{tabular}

Fuente: elaboración propia.

Las estrategias que pone en juego el profesor en el aula son generalmente abiertas, desarrollándose dinámicas donde las respuestas de los alumnos se convierten en contenido y se emplean para profundizar en los mismos [CDCA3 $\mathrm{fi}=61.1 \%$ ]. Podemos verlo en esta secuencia donde los propios alumnos van contestando y el profesor usando sus respuestas:

- I-1_CDCA3: Estamos diferenciando entre si se nace o se hace, hay quien nace con unas características y quien las puede adquirir por una situación, pero le sigue afectando orgánicamente, ¿¿no? ¿Quién decía que Nemo tiene un déficit?

- Alumna 1: Aunque tenga la aleta más corta puede hacer cosas igual.

- Alumna 2: Eso es lo mismo que dijimos antes para minusvalía.

- Alumna 3: La minusvalía tiene que ver con un grado.

- Profesor: La minusvalía tiene que ver con un grado, y el déficit con qué tiene que ver. Con una deficiencia que tienes, pero no te impide hacer algo. Y si es un déficit físico, ¿qué es una discapacidad? 
Estas preguntas también se emplean para conocer qué interesa a los alumnos [PDCA3 $\mathrm{fi}=50 \%$ ], aunque no siempre se usan las respuestas para abordar los contenidos a tratar [CDCA2 fi=33.3\%]. Asimismo, aunque en menor medida, se generan situaciones donde se potencian y valoran las respuestas diversas sin que exista una respuesta única prevista [PDCA4 $\mathrm{fi}=41.7 \%$ ] como refleja el tipo de trabajo que desarrollan los alumnos.

I-2_PDCA4: Para las clases, el profesor propone un proyecto de investigación a realizar en grupo.

Estas estrategias propician espacios para la reflexión y la participación de los alumnos, la cual resulta un pilar fundamental para el proceso [ADCA2 fi=92\%]. Hecho que se refleja en algunas de las actividades propuestas en el aula:

I-2_ADCA2: Profesor: pensar una situación discapacitante y proponer una actividad en la que puedan participar todos los alumnos. El profesor pasa por los grupos y utiliza información que se genera en cada uno estableciendo relaciones con los conocimientos que se trabajan durante la asignatura.

Las situaciones descritas reflejan la primacía de las dinámicas abiertas sobre las cerradas. Las estrategias puestas en juego se usan para abordar el conocimiento y conocer qué interesa a los alumnos, aunque es cierto que estos intereses no llegan a provocar la reorganización del proceso inicialmente planificado.

Este tipo de dinámicas favorecen la toma de decisiones de los alumnos y les ayuda en la construcción de un pensamiento crítico, fundamental para abordar los retos que plantea la sostenibilidad.

Los resultados para el eje dialógico Trabajo de aula se presentan en la tabla 10.

\begin{tabular}{|c|c|c|c|}
\hline \multicolumn{4}{|c|}{ TABLA 10. EJE DIALÓGICO TRABAJO DE AULA: INDIVIDUAL-EN GRUPO } \\
\hline EJE DIALÓGICO SECUNDARIO & Agente & INDICADOR & FreCUENCIA RELATIVA \\
\hline \multirow{4}{*}{$\begin{array}{c}\text { Trabajo de aula, } \\
\text { Individual } \leftrightarrow \text { En grupo }\end{array}$} & \multirow{2}{*}{ Profesor } & PTIG3 & $95.6 \%$ \\
\hline & & PTIG4 & $4.4 \%$ \\
\hline & \multirow{2}{*}{ Alumno } & ATIG3 & $90.5 \%$ \\
\hline & & ATIG4 & $9.5 \%$ \\
\hline
\end{tabular}

Fuente: elaboración propia.

Se trabaja tanto individual como grupalmente potenciándose el trabajo en equipo [PTIG3 fi=95.65\%]:

I-2_PTIG3: Con el puzle de Aronson el profesor pretende proporcionar una experiencia de trabajo cooperativo antes de comenzar los trabajos de investigación de la asignatura. 
Se ha detectado la relevancia del trabajo en grupo [ATIG3 fi=90.5\%], como podemos inferir de algunos ejemplos:

- I-1_ATIG3: «Un pequeño repaso antes de terminar del trabajo que habéis hecho en grupo. Por aquí:

- Alumna: Nos quedan aún las actividades y terminar el cuento.

- Profesor: Pues dadle cańa, que os vais a quedar atrancadillas...».

Otro ejemplo en este sentido:

I-1_ATIG3: Antes de venir a clase, acordaos de iros al diario colaborativo y leer en qué punto estamos. Le echáis un vistazo, porque es una herramienta hecha por vosotras.

El trabajo en grupo es una estrategia empleada para abordar el conocimiento, discutirlo y construirlo conjuntamente. Vertebra el trabajo de aula, es significativo y se combina con el trabajo individual, complementándose ambos. Se tienen en cuenta tanto los intereses individuales como los grupales. Se recrean así escenarios reales de equipos de trabajo en los cuales se ponen en juego valores y conocimientos. tabla 11:

Las frecuencias obtenidas en relación con la Evaluación se presentan en la

\begin{tabular}{|c|c|c|c|}
\hline \multicolumn{4}{|c|}{ TABLA 11. EJE DIALÓGICO EVALUACIÓN: ACREDITATIVA-PROCESUAL } \\
\hline EJE DIALÓGICO SECUNDARIO & Agente & INDICADOR & Frecuencia relativa \\
\hline Evaluación, Acreditativa $\leftrightarrow$ Procesual & Profesor & PEAP3 & $100 \%$ \\
\hline
\end{tabular}

El profesor utiliza distintos instrumentos de evaluación durante el proceso. Asimismo, el alumnado toma parte en esta tarea como coevaluador y decide sobre algunos criterios de evaluación [PEAP3 fi=100\%]. Este ejemplo refleja la elaboración de un diario colaborativo entre toda la clase:

I-2_PEAP3: Durante las clases el profesor negocia con los alumnos el peso que tendrá cada una de las pruebas de evaluación que se desarrollarán en la asignatura.

La situación observada refleja una evaluación inclinada hacia la vertiente procesual, si bien es cierto que la evaluación es el único elemento metodológico analizado cuyos resultados se ciñen a un solo indicador. Aun así podemos decir que está cercana a la que proponemos como favorecedora de la inclusión de la sostenibilidad.

Esta aproximación a través de los diferentes agentes y ejes dialógicos permite describir el proceso de enseñanza-aprendizaje desarrollado y establece los cimientos para la segunda fase de análisis. 
Una vez descrito el proceso de enseñanza-aprendizaje es posible conocer el grado de integración de la sostenibilidad en el aula. Para ello, seleccionamos los resultados más representativos, aquellos con frecuencias fi $\geq 50 \%$, los cuales clasificamos en los diferentes estadios simple, inicial, medio, avanzado y complejo en la figura 2.

Los indicadores de mayor frecuencia se disponen a lo largo de los cinco estadios de integración de sostenibilidad, simple [PCET1 fi $=60.8 \%$, PRNI1 fi $=52.4 \%$, ARNI1 fi $=56 \%$, CRNI1 $\mathrm{fi}=50 \%$ y PRIE1 $\mathrm{fi}=55.6 \%$ ], inicial [PRVH2 $\mathrm{fi}=50 \%$ ], medio [CRNI2 $\mathrm{fi}=50 \%$ y ADCA $2 \mathrm{fi}=92 \%$ ], avanzado [PEAP3 $\mathrm{fi}=100 \%$, PDCA3 $\mathrm{fi}=50 \%$, PTIG3 $\mathrm{fi}=95.6 \%$ y ATIG3 $\mathrm{fi}=90.5 \%$ ] y complejo $[\mathrm{ARVH} 5 \mathrm{fi}=51.7 \%$, CRVH3 $\mathrm{fi}=77.4 \%$, CRIE3 $\mathrm{fi}=50 \%$, y CDCA3 $\mathrm{fi}=61.1 \%$ ], concentrándose en los estadios simple y complejo el mayor número de indicadores.

Los ejes dialógicos que están en el estadio simple y que comprenden a alguno de los tres agentes: profesor, alumno, contenidos, son Competencias: Específicas $\leftrightarrow$ Transversales; Realidad socioambiental: No integrada $\leftrightarrow$ Integrada y Recursos: Internos $\leftrightarrow$ Externos. Sin embargo, para el estadio complejo los elementos metodológicos son Relación profesor-alumno: Vertical $\leftrightarrow$ Horizontal; Dinámicas de aula: Cerradas $\leftrightarrow$ Abiertas y Recursos: Internos $\leftrightarrow$ Externos. Resultan llamativas las dos situaciones extremas en relación con el grado de inclusión de sostenibilidad en el caso de recursos, pues para el agente profesor se encuentra en estadio simple [PRIE1 $\mathrm{fi}=55.6 \%$ ], mientras que para los contenidos en el complejo [CRIE3 $\mathrm{fi}=50 \%$ ]. Es destacable, además, que aquellos elementos metodológicos en que, sin llegar a estar totalmente integrada la sostenibilidad, esta tiene una fuerte presencia. Así, la consideración del papel del agente profesor es significativa en los ejes de evaluación, trabajo en grupo y dinámicas de aula; y el trabajo en grupo para el agente alumno. Mientras que para los agentes alumno y profesor son las dinámicas y el trabajo de aula los ejes que reflejan decisiones más cercanas a una significativa integración de la sostenibilidad en la práctica.

Si nos detenemos en los resultados a nivel global podemos decir que el grado de inclusión de la sostenibilidad en la práctica del sujeto analizado es elevado.

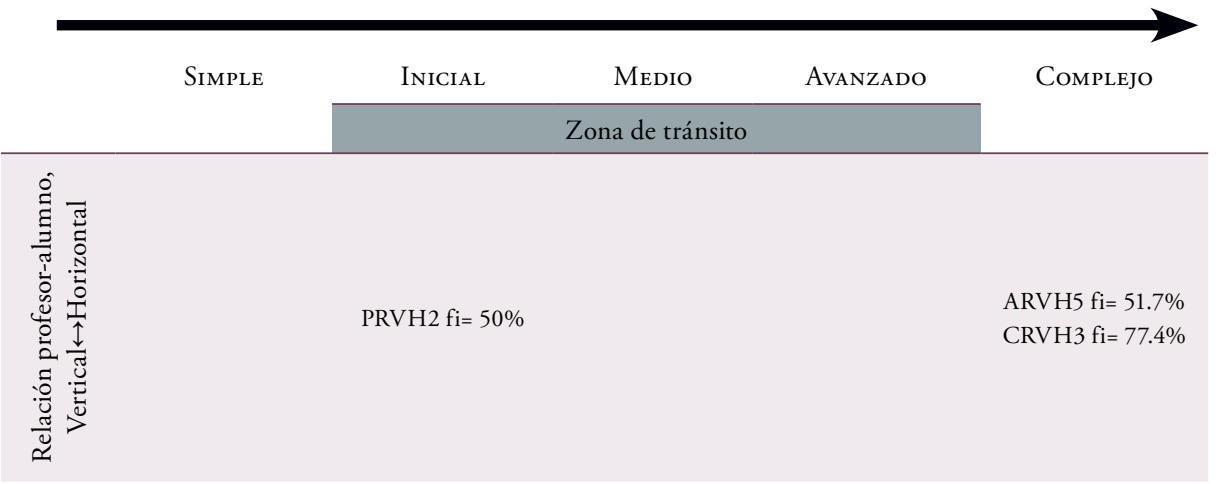




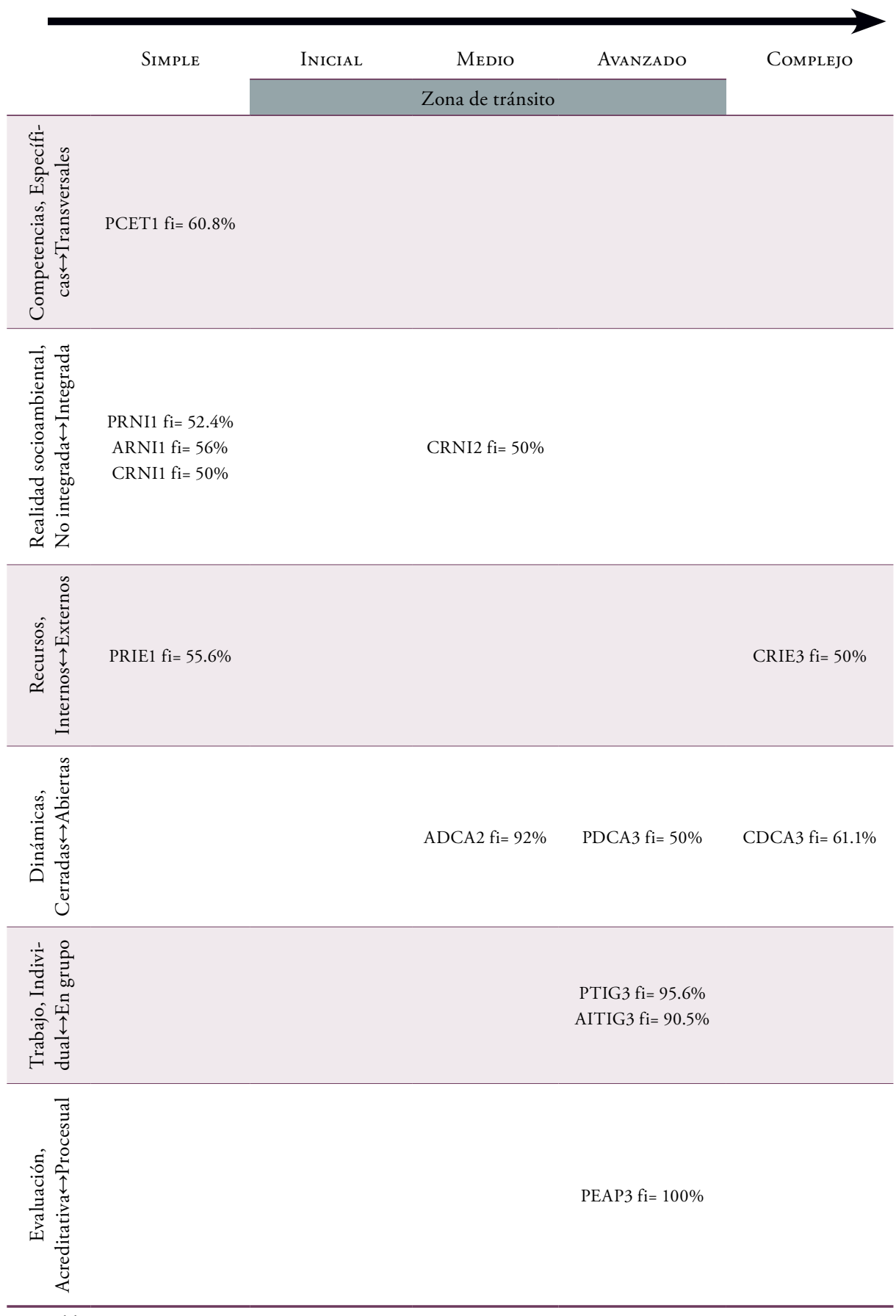

Fuente: elaboración propia.

Figura 2. Grado de inclusión de la sostenibilidad en el aula. 


\section{CONCLUSIONES Y PROSPECTIVA}

Las conclusiones de esta investigación permiten caracterizar la práctica docente y la gradación de la sostenibilidad en sus actuaciones.

En relación con la caracterización, la investigación realizada describe un proceso de enseñanza-aprendizaje donde las prácticas docentes puestas en juego promueven desde varios elementos metodológicos la sostenibilidad, esta tiene una presencia notable en la intervención del sujeto analizado. Se trata de un proceso planificado por el profesor, con claras miras hacia el desarrollo y formación de los futuros maestros, a los que se les conceden claros espacios para la toma de decisiones.

Los aspectos que más se resisten a la inclusión de la sostenibilidad son por un lado las competencias, más centradas en las específicas, y por otro la realidad socioambiental, como refleja la baja integración de la misma para el agente contenidos. Ambos aspectos están próximos a la disciplina. No obstante, las dinámicas utilizadas por el profesor para abordar los contenidos son signo evidente de la inclusión de la sostenibilidad en el proceso, un ejemplo de ello es la relevancia del trabajo en grupo para el funcionamiento de las clases. Cuestiones a las que se suma el modo como el profesor concibe la evaluación en el aula, poniendo en juego diversidad de instrumentos y con los alumnos como agentes evaluados y evaluadores. Hechos que denotan un papel activo del alumno, además de la importancia de recabar información de diferentes fuentes e instrumentos para configurar la imagen final de la evaluación.

Con las características expuestas, consideramos que este profesor está cercano, en varios aspectos, a las prácticas propuestas como impulsoras de la sostenibilidad en el aula. Avanzar hacia un escenario de mayor integración de la sostenibilidad conllevaría desplazar la posición de este profesor hacia una perspectiva más facilitadora de la participación de los alumnos, pues se ha detectado que, indirectamente, detenta un papel de liderazgo excesivo. Esto, sumado a otras cuestiones, podría facilitar que los alumnos aportasen más de su propia cosecha al proceso, pues si bien es cierto que la participación de los estudiantes es estructural para su funcionamiento, no llegan a intervenir de forma autónoma. De otra parte, sería conveniente que las estrategias metodológicas promovieran en mayor medida las competencias transversales y la integración de la realidad socioambiental vinculada al conocimiento disciplinar; por último, habría que incluir en el proceso más recursos externos.

Asimismo, su disciplina, aunque vinculada con mayor intensidad al ámbito social, si se enfoca desde una perspectiva más amplia, puede ser un vector de la inclusión de otros ámbitos de la sostenibilidad, pues el concepto de diversidad, eje de la asignatura, puede ser un punto de partida ya que encierra un gran potencial. Este comprende en sí mismo diálogo entre heterogéneo y homogéneo, incluye que los individuos se entienden a partir de lo que poseen de iguales y al mismo tiempo a partir de lo que los distingue, idea que puede hacerse extensible a otros ámbitos.

De otra parte, como apuntan Cebrián y Junyent (2014), los futuros maestros deberían adquirir durante su formación inicial las competencias profesionales que los preparen para afrontar los diferentes retos de sostenibilidad que se les plantearán durante su ejercicio profesional. Sin embargo, esta formación competencial no será posible si no se trabaja en el aula desde una perspectiva sostenible; es imprescindi- 
ble que el profesorado practique con el ejemplo, más aún en este caso en el que el profesor universitario se convierte en un referente para los maestros en formación.

En otro orden de cosas, la investigación realizada apunta claramente a la necesidad de repensar y reconceptualizar las metodologías docentes universitarias, lo cual desde nuestra perspectiva reclama procesos formativos y de discusión destinados a la comunidad docente. Es un desafío que debemos abordar desde la comunidad científica.

Consideramos que toda investigación en educación debe resultar de utilidad para otros docentes e investigadores (De la Herrán Gascón, 2005). En este sentido y derivado del proceso de investigación, aunque no era un objetivo inicial, consideramos que nuestro estudio aporta un instrumento valioso para el estudio de la práctica docente, como es la HAMS. Esta herramienta ha permitido analizar en profundidad la realidad metodológica del aula estudiada. A través de la herramienta se han identificado debilidades y fortalezas del proceso de enseñanza-aprendizaje objeto de estudio, hecho que revela información sobre pasos a seguir si se pretende avanzar hacia una mayor integración de la sostenibilidad.

La HAMS puede servir como punto de partida para nuevas investigaciones en el ámbito de la ES universitaria. Asimismo, resulta de utilidad al profesorado universitario para reflexionar sobre su práctica docente, esto es, puede constituirse en un potente medio de autodiagnóstico metodológico para aquel profesorado interesado en integrar la sostenibilidad en sus aulas o que se aproxima a este campo de conocimiento.

En este sentido, este estudio abre nuevas y diversas posibilidades de investigación. Las líneas metodológicas que presentamos en la fundamentación teórica e integradas en las HAMS pueden ser un punto de partida para explorar las necesidades formativas del profesorado universitario y diseñar procesos que faciliten la sostenibilización de sus materias. Es una herramienta concebida desde un carácter abierto y flexible que puede concretarse con las particularidades de cada disciplina.

A modo de cierre, la universidad debe responder a las sensibilidades de su tiempo y entorno, constituir un instrumento de desarrollo, de creatividad, de transmisión de valores y transformación social; trabajar desde metodologías que persigan la sostenibilidad dentro de sus aulas puede contribuir a esta labor. Nuestra investigación es un apoyo en esta línea. 


\section{REFERENCIAS BIBLIOGRÁFICAS}

Álvarez, P. (2012). «Los planes institucionales de tutoría y el desarrollo de competencias en el marco del Espacio Europeo de Educación Superior». Perfiles Educativos, XXXIV (137), 28-45.

Aznar, P., Ull, A., Piñero, A. y Martínez-Agut, M.P. (2013). «La sostenibilidad en la formación universitaria: desafíos y oportunidades». Educación XX1, 17 (1), 133-158.

Barrón, A., Navarrete, A. y Ferrer-Balas, D. (2010). «Sostenibilización curricular en las universidades españolas. ¿Ha llegado la hora de actuar?». Revista Eureka sobre Enseñanza y Divulgación de las Ciencias, 7, N. ${ }^{\circ}$ Extr, 388-399.

Bisquerra, R. (1989). «Metodología de recogida de datos». En R. Bisquerra (ed.), Métodos de investigación educativa: guia práctica (pp. 87-122). Barcelona: Ediciones CEAC.

Bonil, J., Calafell, G., Granados, J., Junyent, M. y Tarín, M.R. (2012). «Un modelo formativo para avanzar en la ambientalización curricular». Profesorado. Revista de Curriculum $Y$ Formación de Profesorado, 16 (2), 145-163.

Breinting, S. (1997). «Hacia un nuevo concepto de Educación Ambiental». Carpeta Informativa Del Ceneam, 1-8. Retrieved from http://www.magrama.gob.es/es/ceneam/articulos-de-opinion/1997soren-breiting_tcm7-186955.pdf.

CADEP-CRUE (2012). Directrices para la introducción de la Sostenibilidad en el Curriculum. Actualización de la declaración institucional aprobada en 2005. Retrieved from http:// www.crue.org/Sostenibilidad/CADEP/Documents/DIRECTRICES SOSTENIBILIDAD CRUE 2012.pdf.

Cardeñoso, J.M., Azcárate, P. y Oliva, J.M. (2013). «La sostenibilidad en la formación inicial del profesorado de Secundaria: incidencia en los estudiantes de Ciencias y Matemáticas». Revista Eureka sobre Enseñanza y Divulgación de las Ciencias, 10 (N. . Extra), 780-796.

Cebrián, G., y Junyent, M. (2014). «Competencias profesionales en Educación para la Sostenibilidad: un estudio exploratorio de la visión de futuros maestros». Enseñanza de Las Ciencias, 32.1, 29-49.

Coll, C. (1994). «De qué hablamos cuando hablamos de constructivismo». Cuadernos de Pedagogía, $221,8-12$.

Cotton, D., Warren, M., Maiboroda, O. y Bauley, I. (2007). «Sustainable development, higher education and pedagogy: a study of lecturers' beliefs and attitudes». Environmental Education Research, 13(5), 579-597.

De la Herrán Gascón, A. (2005). «Los grandes paradigmas científicos». En A. de la Herrán Gascón, E. Hashimoto, y E. Machado (eds.), Investigar en Educación. Fundamentos, aplicación y nuevas perspectivas (pp. 381-394). Madrid: Editorial Dilex, S.L.

FLOR, J.I. (2002). «Hacia una visión sistémico-compleja de la problemática ambiental y de la educación». En J. Flor y M. Novo (eds.), Globalización, crisis ambiental y educación (pp. 113-139). Madrid: Secretaría General Técnica MEC.

García-González, E., Jiménez-Fontana, R., Azcárate, P. y Cardeñoso, J. M. (2016). «Inclusion of Sustainability in University Classrooms Through Methodology». In W. Leal Filho, L. Brandli, J. Newman, \& P. Castro (eds.), Handbook of Theory and Practice of Sustainable Development in Higher Education. Berlín: Springer. 
García-González, E., Jiménez-Fontana, R. y Navarrete, A. (2017). «Educar para la sostenibilidad desde las Ciencias Económicas». Revista Iberoamericana de Educación, 73, 155-178.

García-González, E., Jiménez-Fontana, R., Navarrete, A. y Azcárate, P. (2015). «La metodología docente como estrategia para promover la sostenibilidad en las aulas universitarias. Un estudio de caso en la Universidad de Cádiz». Foro de Educación, 13(19), 85-124.

García, B.; Ponce, S., García, H., Caso, J., Morales, C., Martínez, Y., Serna, A., Islas, D., Martínez, S. y Aceves, Y. (2016). «Las competencias del tutor universitario: una aproximación a su definición desde la perspectiva teórica y de la experiencia de sus actores». Perfiles Educativos, XXXVIII (151), 104-122.

García, J. E. (2002). «Los problemas de la Educación Ambiental: ¡̨es posible una Educación Ambiental integradora?». Investigación en la Escuela, 46, 5-25.

García, J.E., Luna, M., Jiménez, R. y Wamba, A.M. (1999). «El análisis de la intervención en el aula: instrumentos y ejemplificaciones». Investigación en la escuela, 39, 63-87.

Hernández, R., Fernández, C. y Baptista, P. (2008). «Similitudes y diferencias entre los enfoques cuantitativo y cualitativo». En Metodología de la investigación (pp. 3-29). México: McGraw-Hil.

Leal Filho, W. (2009). «La educación para la sostenibilidad: iniciativas internacionales». Revista de Educación, número ext, 263.277.

LEFF, E. (2006). «Complejidad, racionalidad ambiental y diálogo de saberes». Carpetas Informativas Del CENEAM, 1-7. Retrieved from http://www.magrama.gob.es/es/ceneam/articulos-de-opinion/2006_01eleff_tcm7-53048.pdf.

Litwin, M.S. (1995). How to measure survey reliability and validity. London: Sage.

Mata-Benito, P., Ballesteros-Velázquez, B. y Padilla-Carmona, M.T. (2013). "Ciudadanía participativa y transformadora: análisis de discursos y propuestas de aprendizaje». Teoría de la Educación, 25 (2), 49-68.

Michavilla, F. (2005). «No sin los profesores». Revista de Educación, 337, 37-49.

Morin, E. (1994). Introducción al pensamiento complejo. Barcelona: Gedisa.

Morin, E. (2008). On complexity. Cresskill: Hampton Press.

Porlán, R. y Rivero, A. (1998). El conocimiento de los profesores. Sevilla: Díada.

Puig, J., Echarri, F. y CASAs, M. (2014). «Educación ambiental, inteligencia espiritual y naturaleza». Teoría de La Educación, 26 (2), 115-140. http://doi.org/10.14201/teoredu2014261115140.

Reyero, D. (2014). «La excelencia docente universitaria. Análisis y propuestas para una mejor evaluación del profesorado universitario». Educación XXI, 17(2), 125-143. https://doi. org/10.5944/educxx1.17.2.11482.

Rieckmann, M. (2012). «Future-oriented higher education: Which key competencies should be fostered through university teaching and learning?». Futures, 44 (2), 127-135. http://doi. org/10.1016/j.futures.2011.09.005.

Rodríguez Izquierdo, R.M. (2007). «Mejora continua de la práctica docente universi taria: una experiencia desde el proceso de convergencia del Espacio Europeo de Educación Superior». REIFOP, 10 (1), 4. Retrieved from http://www.aufop.com/aufop/uploaded_files/ articulos/1224596123.pdf.

Ruiz Ortega, F.J. (2007). «Modelos didácticos para la enseñanza de las ciencias». Revista Latinoamericana de Estudios Educativos, 3 (2), 41-60. 
Sanmartí, N. (2007). 10 ideas clave. Evaluar para aprender. Barcelona: Graò.

Stake, R. (2005). «Qualitative case studies». In N. Denzin (ed.), The Sage Handbook of Qualitative Research (pp. 273-285). London: Sage.

Thомаs, I. (2016). «Challenges for implementation of education for sustainable development in higher education institutions». In M. Barth, G. Michelsen, M. Rieckmann, y I. Thomas (eds.), Routledge Handbook of Higher Education for Sustainable Development (pp. 56-71). London, New York: Routledge.

UNESCO. (2015). «Declaración de Incheon». In Foro Mundial sobre la Educación 2015. Incheon.

Viladot, P. y Pedreira, M. (2012). «Fer bones pràctiques als musèus i centres de ciències». In J. Bonil, T. Gómez, M. Pejó, y P. Viladot (eds.), Som educaciò. Ensenyar i aprende als mueseus i centre de ciència: una propuesta de model didàctic (pp. 37-71). Barcelona. Retrieved from http://issuu.com/museuciencies/docs/manuals_del_museu_vol_1__2012.

Vilches, A. y Gil Pérez, D. (2012). «La educación para la sostenibilidad en la Universidad: el reto de la formación del profesorado». Profesorado. Revista de Currículum y Formación de Profesorado, 16 (2), 25-43.

Vilches, A. y Gil PéRez, D. (2016). «La transición a la sostenibilidad como objetivo urgente para la superación de la crisis sistémica actual». Revista Eureka Sobre Enseñanza Y Divulgación de Las Ciencias, 13 (2), 395-407.

WALKER, V.S. (2016). «El trabajo docente en la universidad: condiciones, dimensiones y tensiones». Perfiles Educativos, XXXVIII (153), 105-119.

WALs, A.E.J. y JickLING, B. (2002). “Sustainability” in higher education». International Journal of Sustainability in Higher Education, 3(3), 221-232. http://doi.org/10.1108/14676370210434688.

Warburton, K. (2003). «Deep learning and education for sustainability». International Journal of Sustainability in Higher Education, 4(1), 44-56. https://doi.org/10.1108/14676370310455332.

Wiek, A., Xiong, A., Brundiers, K. y Leeuw, S. van Der. (2014). «Integrating problem- and project-based learning into sustainability programs. A case study on the School of Sustainability at Arizona State University». International Journal of Sustainability in Higher Education, 15 (4), 413-449. 